\title{
Super-de Sitter and alternative super-Poincaré symmetries
}

\author{
V.N. Tolstoy
}

\begin{abstract}
It is well-known that de Sitter Lie algebra $\mathfrak{o}(1,4)$ contrary to anti-de Sitter one $\mathfrak{o}(2,3)$ does not have a standard $\mathbb{Z}_{2}$-graded superextension. We show here that the Lie algebra $\mathfrak{o}(1,4)$ has a superextension based on the $\mathbb{Z}_{2} \times \mathbb{Z}_{2}$-grading. Using the standard contraction procedure for this superextension we obtain an alternative super-Poincaré algebra with the $\mathbb{Z}_{2} \times \mathbb{Z}_{2}$-grading.
\end{abstract}

\section{Introduction}

In supergravity theory (SUGRA) already for more than 20 years there is the following unsolved (up to now) problem. All physical reasonable solutions of SURGA models with cosmological constants $\Lambda$ have been constructed for the case $\Lambda<0$, i.e. for the anti-de Sitter metric

$$
g_{a b}=\operatorname{diag}(1,-1,-1,-1,1), \quad(a, b=0,1,2,3,4)
$$

with the space-time symmetry $\mathfrak{o}(2,3)$. In the case $\Lambda>0$, i.e. for the Sitter metric

$$
g_{a b}=\operatorname{diag}(1,-1,-1,-1,-1), \quad(a, b=0,1,2,3,4)
$$

with the space-time symmetry $\mathfrak{o}(1,4)$ no reasonable solutions have been found. For example, in SUGRA it was obtained the following relation

$$
\Lambda=-3 m^{2}
$$

where $m$ is the massive parameter of gravitinos. Thus if $\Lambda>0$, then $m$ is imaginary.

V.N. Tolstoy

Lomonosov Moscow State University, Skobeltsyn Institute of Nuclear Physics (MSU SINP), 1(2) Leninskie Gory, GSP-1, Moscow 119991, Russian Federation, e-mail: tolstoy@nucl-th.sinp.msu.ru 
In my opinion these problems for the case $\Lambda>0$ are connected with superextensions of anti-de Sitter $\mathfrak{o}(2,3)$ and de Sitter $\mathfrak{o}(1,4)$ symmetries. The $\mathfrak{o}(2,3)$ symmetry has the superextension - the superalgebra osp $(1 \mid(2,3))$. This is the usual $\mathbb{Z}_{2^{-}}$ graded superalgebra. In the case of $\mathfrak{o}(1,4)$ such superextension does not exist. However the Lie algebra $\mathfrak{o}(1,4)$ has an alternative superextension that is based on the $\mathbb{Z}_{2} \times \mathbb{Z}_{2}$-grading and a preliminary analysis shows that we can construct the reasonable SUGRA models for the case $\Lambda>0$. In this paper we shall consider certain $\mathbb{Z}_{2} \times \mathbb{Z}_{2}$-graded supersymmetries, but we will not discuss supergravity models based on such supersymmetries.

All standard relativistic SUSY (super-anti de Sitter, super-Poincaré, super-conformal, extended $N$-supersymmetry, etc) are based on usual ( $\mathbb{Z}_{2}$-graded) Lie superalgebras $(\operatorname{osp}(1 \mid(2,3)), \operatorname{su}(N \mid(2,2)), \operatorname{osp}(N \mid(2,3))$ etc). It turns out that every standard relativistic SUSY has an alternative variant based on an alternative $\left(\mathbb{Z}_{2} \times \mathbb{Z}_{2}\right.$-graded $)$ Lie superalgebra:

\section{Standard relativistic SUSY $\rightleftarrows$ Alternative relativistic SUSY}

Distinctive features of the standard and alternative relativistic symmetries (in the example of Poincaré SUSY) are connected with the relations between the fourmomenta and the $Q$-charges and also between the space-time coordinates and the Grassmann variables. Namely, we have.

(I) For the standard ( $\mathbb{Z}_{2}$-graded) Poincaré SUSY:

$$
\begin{aligned}
{\left[P_{\mu}, Q_{\alpha}\right] } & =\left[P_{\mu}, \bar{Q}_{\dot{\alpha}}\right]=0, \quad\left\{Q_{\alpha}, \bar{Q}_{\dot{\beta}}\right\}=2 \sigma_{\alpha \dot{\beta}}^{\mu} P_{\mu}, \\
{\left[x_{\mu}, \theta_{\alpha}\right] } & =\left[x_{\mu}, \dot{\theta}_{\dot{\alpha}}\right]=\left\{\theta_{\alpha}, \bar{\theta}_{\dot{\beta}}\right\}=0 .
\end{aligned}
$$

(II) For the altrenative $\left(\mathbb{Z}_{2} \times \mathbb{Z}_{2}\right.$-graded) Poincaré SUSY:

$$
\begin{aligned}
& \left\{P_{\mu}, Q_{\alpha}\right\}=\left\{P_{\mu}, \bar{Q}_{\dot{\alpha}}\right\}=0, \quad\left[Q_{\alpha}, \bar{Q}_{\dot{\beta}}\right]=2 \sigma_{\alpha \dot{\beta}}^{\mu} P_{\mu}, \\
& \left\{x_{\mu}, \theta_{\alpha}\right\}=\left\{x_{\mu}, \dot{\theta}_{\dot{\alpha}}\right\}=\left[\theta_{\alpha}, \bar{\theta}_{\dot{\beta}}\right]=0 .
\end{aligned}
$$

We wrote down only the relations which are changed in the $\mathbb{Z}_{2}$ - and $\mathbb{Z}_{2} \times \mathbb{Z}_{2}$-cases.

The paper is organized as follows. Section 2 provides definitions and general structure of $\mathbb{Z}_{2}$ - and $\mathbb{Z}_{2} \times \mathbb{Z}_{2}$-graded superalgebras and also some classification of such simple Lie superalgebras. In Section 3 we describe the orthosymplectic $\mathbb{Z}_{2^{-}}$ and $\mathbb{Z}_{2} \times \mathbb{Z}_{2}$-graded superalgebras $\mathfrak{o s p}(1 \mid 4)$ and $\mathfrak{o s p}(1 \mid 2,2)$ and their real forms. We show here that a real form of $\mathfrak{o s p}(1 \mid 4)$ contains $\mathfrak{o}(2,3)$ and a real form of $\mathfrak{o s p}(1 \mid 2,2)$ contains $\mathfrak{o}(1,4)$. In Section 4 using the standard contraction procedure for the superextension osp $(1 \mid 2,2)$ we obtain an alternative super-Poincaré algebra with the $\mathbb{Z}_{2} \times \mathbb{Z}_{2}$-grading. 


\section{$2 \mathbb{Z}_{2-}$ and $\mathbb{Z}_{2} \times \mathbb{Z}_{2}$-graded Lie superalgebras}

$A \mathbb{Z}_{2}$-graded superalgebra [1]. A $\mathbb{Z}_{2}$-graded Lie superalgebra (LSA) $\mathfrak{g}$, as a linear space, is a direct sum of two graded components

$$
\mathfrak{g}=\bigoplus_{a=0,1} \mathfrak{g}_{a}=\mathfrak{g}_{0} \oplus \mathfrak{g}_{1}
$$

with a bilinear operation (the general Lie bracket), $[[\cdot, \cdot]]$, satisfying the identities:

$$
\begin{aligned}
\left.\operatorname{deg}\left(\left[x_{a}, y_{b}\right]\right]\right) & =\operatorname{deg}\left(x_{a}\right)+\operatorname{deg}\left(y_{b}\right)=a+b \quad(\bmod 2), \\
{\left.\left[x_{a}, y_{b}\right]\right] } & \left.=-(-1)^{a b}\left[y_{b}, x_{a}\right]\right], \\
{\left[\left[x_{a},\left[\left[y_{b}, z\right]\right]\right]\right.} & \left.=\left[\left[\left[x_{a}, y_{b}\right]\right], z\right]\right]+(-1)^{a b}\left[\left[y_{b},\left[\left[x_{a}, z\right]\right]\right],\right.
\end{aligned}
$$

where the elements $x_{a}$ and $y_{b}$ are homogeneous, $x_{a} \in \mathfrak{g}_{a}, y_{b} \in \mathfrak{g}_{b}$, and the element $z \in \mathfrak{g}$ is not necessarily homogeneous. The grading function $\operatorname{deg}(\cdot)$ is defined for homogeneous elements of the subspaces $\mathfrak{g}_{0}$ and $\mathfrak{g}_{1}$ modulo $2, \operatorname{deg}\left(\mathfrak{g}_{0}\right)=0$, $\operatorname{deg}\left(\mathfrak{g}_{1}\right)=1$. The first identity $(9)$ is called the grading condition, the second identity (10) is called the symmetry property and the condition (11) is the Jacobi identity. It follows from (9) that $\mathfrak{g}_{0}$ is a Lie subalgebra in $\mathfrak{g}$, and $\mathfrak{g}_{1}$ is a $\mathfrak{g}_{0}$-module. It follows from (9) and (10) that the general Lie bracket [[., $\cdot]$ for homogeneous elements posses two values: commutator $[\cdot, \cdot]$ and anticommutator $\{\cdot, \cdot\}$.

$A \mathbb{Z}_{2} \times \mathbb{Z}_{2}$-graded superalgebra [4]. A $\mathbb{Z}_{2} \times \mathbb{Z}_{2}$-graded LSA $\tilde{\mathfrak{g}}$, as a linear space, is a direct sum of four graded components

$$
\tilde{\mathfrak{g}}=\bigoplus_{\mathbf{a}=\left(a_{1}, a_{2}\right)} \tilde{\mathfrak{g}}_{\mathbf{a}}=\tilde{\mathfrak{g}}_{(0,0)} \oplus \tilde{\mathfrak{g}}_{(1,1)} \oplus \tilde{\mathfrak{g}}_{(1,0)} \oplus \tilde{\mathfrak{g}}_{(0,1)}
$$

with a bilinear operation $[[\cdot, \cdot]$ satisfying the identities (grading, symmetry, Jacobi):

$$
\begin{aligned}
\operatorname{deg}\left(\left[x_{\mathbf{a}}, y_{\mathbf{b}}\right]\right) & =\operatorname{deg}\left(x_{\mathbf{a}}\right)+\operatorname{deg}\left(y_{\mathbf{b}}\right)=\mathbf{a}+\mathbf{b}=\left(a_{1}+b_{1}, a_{2}+b_{2}\right), \\
{\left[\left[x_{\mathbf{a}}, y_{\mathbf{b}}\right]\right] } & =-(-1)^{\mathbf{a} b}\left[\left[y_{\mathbf{b}}, x_{\mathbf{a}}\right],\right. \\
{\left[\left[x_{\mathbf{a}},\left[\left[y_{\mathbf{b}}, z\right]\right]\right]\right.} & \left.\left.=\llbracket\left[\left[x_{\mathbf{a}}, y_{\mathbf{b}}\right]\right], z\right]\right]+(-1)^{\mathbf{a b}}\left[\left[y_{\mathbf{b}}, \llbracket\left[x_{\mathbf{a}}, z\right]\right]\right],
\end{aligned}
$$

where the vector $\left(a_{1}+b_{1}, a_{2}+b_{2}\right)$ is defined $\bmod (2,2)$ and $\mathbf{a b}=a_{1} b_{1}+a_{2} b_{2}$. Here in (13)-(15) $x_{\mathbf{a}} \in \tilde{\mathfrak{g}}_{\mathbf{a}}, y_{\mathbf{b}} \in \tilde{\mathfrak{g}}_{\mathbf{b}}$, and the element $z \in \tilde{\mathfrak{g}}$ is not necessarily homogeneous. It follows from (13) that $\tilde{\mathfrak{g}}_{(0,0)}$ is a Lie subalgebra in $\tilde{\mathfrak{g}}$, and the subspaces $\tilde{\mathfrak{g}}_{(1,1)}$, $\tilde{\mathfrak{g}}_{(1,0)}$ and $\tilde{\mathfrak{g}}_{(0,1)}$ are $\tilde{\mathfrak{g}}_{(0,0)}$-modules. It should be noted that $\tilde{\mathfrak{g}}_{(0,0)} \oplus \tilde{\mathfrak{g}}_{(1,1)}$ is a Lie subalgebra in $\tilde{\mathfrak{g}}$ and the subspace $\tilde{\mathfrak{g}}_{(1,0)} \oplus \tilde{\mathfrak{g}}_{(0,1)}$ is a $\tilde{\mathfrak{g}}_{(0,0)} \oplus \tilde{\mathfrak{g}}_{(1,1)}$-module, and moreover $\left\{\tilde{\mathfrak{g}}_{(1,1)}, \tilde{\mathfrak{g}}_{(1,0)}\right\} \subset \tilde{\mathfrak{g}}_{(0,1)}$ and vice versa $\left\{\tilde{\mathfrak{g}}_{(1,1)}, \tilde{\mathfrak{g}}_{(0,1)}\right\} \subset \tilde{\mathfrak{g}}_{(1,0)}$. It follows from (13) and (14) that the general Lie bracket $[[\cdot, \cdot]]$ for homogeneous elements posses two values: commutator $[\cdot, \cdot]$ and anticommutator $\{\cdot, \cdot\}$ as well as in the previous $\mathbb{Z}_{2}$-case. 
Let us introduce a useful notation of parity of homogeneous elements: the parity $p(x)$ of a homogeneous element $x$ is a scalar square of its grading $\operatorname{deg}(x)$ modulo 2. It is evident that for the $\mathbb{Z}_{2}$-graded superalgebra $\mathfrak{g}$ the parity coincides with the grading: $p\left(\mathfrak{g}_{a}\right)=\operatorname{deg}\left(\mathfrak{g}_{a}\right)=\bar{a}(\bar{a}=\overline{0}, \overline{1}) \sqrt{1}$. In the case of the $\mathbb{Z}_{2} \times \mathbb{Z}_{2}$-graded superalgebra $\tilde{\mathfrak{g}}$ we have

$$
p\left(\tilde{\mathfrak{g}}_{\mathbf{a}}\right):=\mathbf{a}^{2}=a_{1}^{2}+a_{2}^{2} \quad(\bmod 2),
$$

that is

$$
p\left(\tilde{\mathfrak{g}}_{(0,0)}\right)=p\left(\tilde{\mathfrak{g}}_{(1,1)}\right)=\overline{0}, \quad p\left(\tilde{\mathfrak{g}}_{(1,0)}\right)=p\left(\tilde{\mathfrak{g}}_{(0,1)}\right)=\overline{1} .
$$

Homogeneous elements with the parity $\overline{0}$ are called even and with parity $\overline{1}$ are odd. Thus,

$$
\tilde{\mathfrak{g}}=\tilde{\mathfrak{g}}_{0} \oplus \tilde{\mathfrak{g}}_{\overline{1}}, \quad \tilde{\mathfrak{g}}_{\overline{0}}=\tilde{\mathfrak{g}}_{(0,0)} \oplus \tilde{\mathfrak{g}}_{(1,1)}, \quad \tilde{\mathfrak{g}}_{\overline{1}}=\tilde{\mathfrak{g}}_{(1,0)} \oplus \tilde{\mathfrak{g}}_{(0,1)} .
$$

The even subspace $\tilde{\mathfrak{g}}_{\overline{0}}$ is a subalgebra and the odd one $\tilde{\mathfrak{g}}_{\overline{1}}$ is a $\tilde{\mathfrak{g}}_{\overline{0}}$-module. Thus the parity unifies "cousinly" the $\mathbb{Z}_{2}$ - and $\mathbb{Z}_{2} \times \mathbb{Z}_{2}$-graded superalgebras.

Classification of the $\mathbb{Z}_{2}$ - and $\mathbb{Z}_{2} \times \mathbb{Z}_{2}$-graded simple Lie superalgebras. A complete list of simple $\mathbb{Z}_{2}$-graded (standard) Lie superalgebras was obtained by Kac [1]. The following scheme resumes the classification [2]:

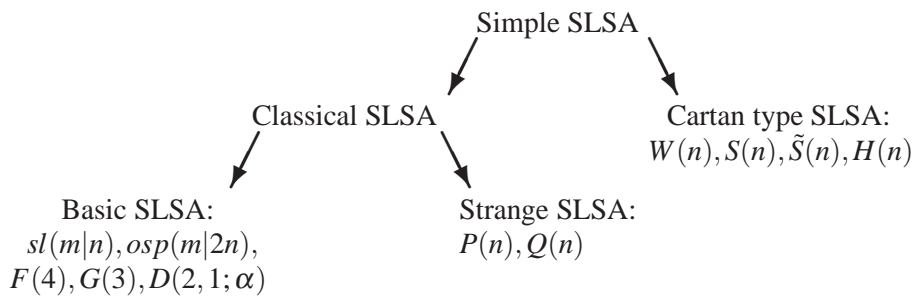

There is a $\mathbb{Z}_{2} \times \mathbb{Z}_{2}$-analog (alternative superalgebras) of this scheme:

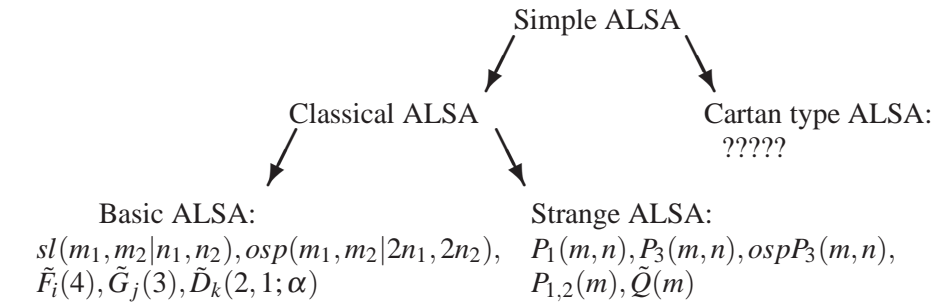

where $i=1,2, \ldots, 6, j=1,2,3, k=1,2,3$. It should be noted that the classification of the classical series $s l\left(m_{1}, m_{2} \mid n_{1}, n_{2}\right)$, osp $\left(m_{1}, m_{2} \mid 2 n_{1}, 2 n_{2}\right)$ and all strange series was obtain by Rittenberg and Wyler in [4].

\footnotetext{
${ }^{1}$ Integer value of the parity will be denoted with the bar.
} 
There are numerous references about the $\mathbb{Z}_{2}$-graded Lie superalgebras and their applications. Unfortunately, in the $\mathbb{Z}_{2} \times \mathbb{Z}_{2}$-case the situation is somewhat poor. There are a few references where some $\mathbb{Z}_{2} \times \mathbb{Z}_{2}$-graded Lie superalgebras were studied and applied [3]-[8].

Analysis of matrix realizations of the basic $\mathbb{Z}_{2} \times \mathbb{Z}_{2}$-graded Lie superalgebras shows that these superalgebras (as well as the $\mathbb{Z}_{2}$-graded Lie superalgebras) have Cartan-Weyl and Chevalley bases, Weyl groups, Dynkin diagrams, etc. However these structures have a specific characteristics for the $\mathbb{Z}_{2}$ - and $\mathbb{Z}_{2} \times \mathbb{Z}_{2}$-graded cases. Let us consider, for example, the Dynkin diagrams. In the case of the $\mathbb{Z}_{2}$-graded superalgebras the nodes of the Dynkin diagram and corresponding simple roots occur at three types:

white $\bigcirc, \quad$ gray $\otimes, \quad$ dark $\bigcirc$

While in the case of $\mathbb{Z}_{2} \times \mathbb{Z}_{2}$-graded superalgebras we have six types of nodes:

$$
\begin{array}{rrr}
\text { (00)-white } \bigcirc, & \text { (11)-white } \oint_{1}, & (10) \text {-gray } \otimes, \\
(01) \text {-gray } \bigotimes, & \quad(10) \text {-dark } \phi, & \text { (01)-dark } \varphi .
\end{array}
$$

In the next Section we consider in detail two basic superalgebras of rank 2: the orthosymplectic $\mathbb{Z}_{2}$-graded superalgebra osp $(1 \mid 4)$ and the orthosymplectic $\mathbb{Z}_{2} \times \mathbb{Z}_{2}$ graded superalgebra osp $(1 \mid 2,2):=\mathfrak{o s p}(1,0 \mid 2,2)$. It will be shown that their real forms, which contain the Lorentz subalgebra $\mathfrak{o}(1,3)$, give us the super-anti-de Sitter (in the $\mathbb{Z}_{2}$-graded case) and super-de Sitter (in the $\mathbb{Z}_{2} \times \mathbb{Z}_{2}$-graded case) Lie superalgebras.

\section{Anti-de Sitter and de Sitter superalgebras}

The orthosymplectic $\mathbb{Z}_{2}$-graded superalgebra osp $(1 \mid 4)$. The Dynkin diagram:

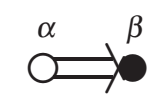

The Serre relations:

$$
\left[e_{ \pm \alpha},\left[e_{ \pm \alpha}, e_{ \pm \beta}\right]\right]=0, \quad\left[\left\{\left[e_{ \pm \alpha}, e_{ \pm \beta}\right], e_{ \pm \beta}\right\}, e_{ \pm \beta}\right]=0 .
$$

The root system $\Delta_{+}$:

$$
\underbrace{2 \beta, 2 \alpha+2 \beta, \alpha, \alpha+2 \beta}_{\operatorname{deg}(\cdot)=0}, \underbrace{\beta, \alpha+\beta}_{\operatorname{deg}(\cdot)=1} .
$$

The orthosymplectic $\mathbb{Z}_{2} \times \mathbb{Z}_{2}$-graded superalgebra osp $(1 \mid 2,2)$. The Dynkin diagram: 
The Serre relations:

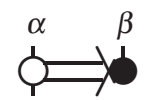

$$
\left\{e_{ \pm \alpha},\left\{e_{ \pm \alpha}, e_{ \pm \beta}\right\}\right\}=0, \quad\left\{\left[\left\{e_{ \pm \alpha}, e_{ \pm \beta}\right\}, e_{ \pm \beta}\right], e_{ \pm \beta}\right\}=0 .
$$

The root system $\Delta_{+}$:

$$
\underbrace{2 \beta, 2 \alpha+2 \beta}_{\operatorname{deg}(\cdot)=(00)}, \underbrace{\alpha, \alpha+2 \beta}_{\operatorname{deg}(\cdot)=(11)}, \underbrace{\beta}_{\operatorname{deg}(\cdot)=(10)}, \underbrace{\alpha+\beta}_{\operatorname{deg}(\cdot)=(01)} .
$$

Commutation relations, which contain Cartan elements, are the same for the $\mathfrak{o s p}(1 \mid 4)$ and $\mathfrak{o s p}(1 \mid 2,2)$ superalgebras and they are:

$$
\begin{aligned}
{\left[\left[e_{\gamma}, e_{-\gamma^{\prime}}\right]\right.} & =\delta_{\gamma, \gamma^{\prime}} h_{\gamma}, \\
{\left[h_{\gamma}, e_{\gamma^{\prime}}\right] } & =\left(\gamma, \gamma^{\prime}\right) e_{\gamma^{\prime}}
\end{aligned}
$$

for $\gamma, \gamma^{\prime} \in\{\alpha, \beta\}$. These relations together with the Serre relations (19) and (21) correspondingly are called the defining relations of the superalgebras osp $(1 \mid 4)$ and $\mathfrak{o s p}(1 \mid 2,2)$ correspondingly. It is easy to see that these defining relations are invariant with respect to the non-graded Cartan involution $\left(^{\dagger}\right)\left(\left(x^{\dagger}\right)^{\dagger}=x,[[x, y]]^{\dagger}=\left[\left[y^{\dagger}, x^{\dagger}\right]\right]\right.$ for any homogenous elements $x$ and $y$ ):

$$
e_{ \pm \gamma}^{\dagger}=e_{\mp \gamma}, \quad h_{\gamma}^{\dagger}=h_{\gamma} .
$$

The composite root vectors $e_{ \pm \gamma}\left(\gamma \in \Delta_{+}\right)$for $\mathfrak{o s p}(1 \mid 4)$ and $\mathfrak{o s p}(1 \mid 2,2)$ are defined as follows

$$
\begin{aligned}
e_{\alpha+\beta} & :=\llbracket\left[e_{\alpha}, e_{\beta} \rrbracket,\right. & e_{\alpha+2 \beta} & :=\llbracket e_{\alpha+\beta}, e_{\beta} \rrbracket, \\
e_{2 \alpha+2 \beta} & :=\frac{1}{\sqrt{2}}\left\{e_{\alpha+\beta}, e_{\alpha+\beta}\right\}, & e_{2 \beta} & :=\frac{1}{\sqrt{2}}\left\{e_{\beta}, e_{\beta}\right\}, \\
e_{-\gamma} & :=e_{\gamma}^{\dagger} . & &
\end{aligned}
$$

These root vectors satisfy the non-vanishing relations: 


$$
\begin{aligned}
{\left[e_{\alpha}, e_{\alpha+2 \beta}\right] } & =(-1)^{\operatorname{deg} \alpha \cdot \operatorname{deg} \beta} \sqrt{2} e_{2 \alpha+2 \beta}, & {\left[e_{\alpha}, e_{2 \beta}\right] } & =\sqrt{2} e_{\alpha+2 \beta}, \\
{\left[e_{\alpha+\beta}, e_{-\alpha}\right] } & =-(-1)^{\operatorname{deg} \alpha \cdot \operatorname{deg} \beta} e_{\beta}, & {\left[e_{\alpha+2 \beta}, e_{-\alpha}\right] } & =-\sqrt{2} e_{2 \beta}, \\
{\left[e_{2 \alpha+2 \beta}, e_{-\alpha}\right] } & =-(-1)^{\operatorname{deg} \alpha \cdot \operatorname{deg} \beta} \sqrt{2} e_{\alpha+2 \beta}, & {\left[e_{2 \beta}, e_{-\beta}\right] } & =-\sqrt{2} e_{\beta}, \\
{\left.\left[e_{\alpha+2 \beta}, e_{-\alpha-\beta}\right]\right] } & =-(-1)^{\operatorname{deg} \alpha \cdot \operatorname{deg} \beta} e_{\beta}, & {\left[e_{\beta}, e_{-\alpha-\beta}\right] } & =e_{-\alpha}, \\
{\left[e_{\beta}, e_{-\alpha-2 \beta} \rrbracket\right.} & =-e_{-\alpha-\beta}, & {\left[e_{2 \alpha+2 \beta}, e_{-\alpha-\beta}\right] } & =-\sqrt{2} e_{\alpha+\beta}, \\
{\left[e_{\alpha+2 \beta}, e_{-2 \alpha-2 \beta}\right] } & =-(-1)^{\operatorname{deg} \alpha \cdot \operatorname{deg} \beta} \sqrt{2} e_{-\alpha}, & {\left[e_{2 \beta}, e_{-\alpha-2 \beta}\right] } & =-\sqrt{2} e_{-\alpha}, \\
\left\{e_{\alpha+\beta}, e_{-\alpha-\beta}\right\} & =h_{\alpha}+h_{\beta}, & {\left[e_{\alpha+2 \beta}, e_{-\alpha-2 \beta}\right] } & =-h_{\alpha}-2 h_{\beta}, \\
{\left[e_{2 \beta}, e_{-2 \beta}\right] } & =-2 h_{\beta}, & {\left[e_{2 \alpha+2 \beta}, e_{-2 \alpha-2 \beta}\right] } & =-2 h_{\alpha}-2 h_{\beta} .
\end{aligned}
$$

The rest of non-zero relations is obtained by applying the operation $\left(^{\dagger}\right)$ to these relations.

Now we find real forms of $\mathfrak{o s p}(1 \mid 4)$ and $\mathfrak{o s p}(1 \mid 2,2)$, which contain the real Lorentz subalgebra $\mathfrak{s o}(1,3)$. It is not difficult to check that the antilinear mapping $\left(^{*}\right)\left(\left(x^{*}\right)^{*}=x,[[x, y]]^{*}=\left[\left[y^{*}, x^{*}\right]\right]\right.$ for any homogenous elements $x$ and $\left.y\right)$ given by

$$
\begin{aligned}
e_{ \pm \alpha}^{*} & =-(-1)^{\operatorname{deg} \alpha \cdot \operatorname{deg} \beta} e_{\mp \alpha}, & e_{ \pm \beta}^{*} & =-i e_{ \pm(\alpha+\beta)}, \\
e_{ \pm 2 \beta}^{*} & =-e_{ \pm(2 \alpha+2 \beta)}, & e_{ \pm(\alpha+2 \beta)}^{*} & =-e_{ \pm(\alpha+2 \beta)}, \\
h_{\alpha}^{*} & =h_{\alpha}, & h_{\beta}^{*} & =-h_{\alpha}-h_{\beta} .
\end{aligned}
$$

is an antiinvolution and the desired real form with respect to the antiinvolution is presented as follows.

The Lorentz algebra $\mathfrak{o}(1,3)$ :

$$
\begin{aligned}
& L_{12}=-\frac{1}{2} h_{\alpha}, \\
& L_{13}=-\frac{i}{2 \sqrt{2}}\left(e_{2 \beta}+e_{2 \alpha+2 \beta}+e_{-2 \beta}+e_{-2 \alpha-2 \beta}\right), \\
& L_{23}=-\frac{1}{2 \sqrt{2}}\left(e_{2 \beta}-e_{2 \alpha+2 \beta}-e_{-2 \beta}+e_{-2 \alpha-2 \beta}\right), \\
& L_{01}=\frac{i}{2 \sqrt{2}}\left(e_{2 \beta}+e_{2 \alpha+2 \beta}-e_{-2 \beta}-e_{-2 \alpha-2 \beta}\right), \\
& L_{02}=\frac{1}{2 \sqrt{2}}\left(e_{2 \beta}-e_{2 \alpha+2 \beta}+e_{-2 \beta}-e_{-2 \alpha-2 \beta}\right), \\
& L_{03}=-\frac{i}{2}\left(h_{\alpha}+2 h_{\beta}\right) .
\end{aligned}
$$


The generators $L_{\mu 4}$ :

$$
\begin{aligned}
L_{04} & =-\frac{i}{2}\left(e_{\alpha+2 \beta}+(-1)^{\operatorname{deg} \alpha \cdot \operatorname{deg} \beta} e_{-\alpha-2 \beta}\right), \\
L_{14} & =-\frac{i}{2}\left(e_{\alpha}+(-1)^{\operatorname{deg} \alpha \cdot \operatorname{deg} \beta} e_{-\alpha}\right), \\
L_{24} & =\frac{1}{2}\left(e_{\alpha}-(-1)^{\operatorname{deg} \alpha \cdot \operatorname{deg} \beta} e_{-\alpha}\right), \\
L_{34} & =-\frac{i}{2}\left(e_{\alpha+2 \beta}-(-1)^{\operatorname{deg} \alpha \cdot \operatorname{deg} \beta} e_{-\alpha-2 \beta}\right) .
\end{aligned}
$$

Here are: $\operatorname{deg} \alpha=0, \operatorname{deg} \beta=1$, i.e. $(-1)^{\operatorname{deg} \alpha \cdot \operatorname{deg} \beta}=1$, for the case of the $\mathbb{Z}_{2}$ grading; $\operatorname{deg} \alpha=(1,1), \operatorname{deg} \beta=(1,0)$, i.e. $(-1)^{\operatorname{deg} \alpha \cdot \operatorname{deg} \beta}=-1$, for the case of the $\mathbb{Z}_{2} \times \mathbb{Z}_{2}$-grading.

The all elements $L_{a b}(a, b=0,1,2,3,4)$ satisfy the relations

$$
\begin{aligned}
{\left[L_{a b}, L_{c d}\right] } & =i\left(g_{b c} L_{a d}-g_{b d} L_{a c}+g_{a d} L_{b c}-g_{a c} L_{b d}\right), \\
L_{a b} & =-L_{b a}, \quad L_{a b}^{*}=L_{a b},
\end{aligned}
$$

where the metric tensor $g_{a b}$ is given by

$$
\begin{aligned}
g_{a b} & =\operatorname{diag}\left(1,-1,-1,-1, g_{44}^{(\alpha)}\right), \\
g_{44}^{(\alpha)} & =(-1)^{\operatorname{deg} \alpha \cdot \operatorname{deg} \beta} .
\end{aligned}
$$

Thus we see that in the case of the $\mathbb{Z}_{2}$-grading, $(-1)^{\operatorname{deg} \alpha \cdot \operatorname{deg} \beta}=1$, the generators (28) and (29) generate the anti-de-Sitter algebra $\mathfrak{o}(2,3)$, and in the case of the $\mathbb{Z}_{2} \times$ $\mathbb{Z}_{2}$-grading, $(-1)^{\operatorname{deg} \alpha \cdot \operatorname{deg} \beta}=-1$, the generators (28) and (29) generate the de-Sitter algebra $\mathfrak{o}(1,4)$.

Finally we introduce the "supercharges":

$$
\begin{array}{ll}
Q_{1}:=\sqrt{2} \exp \left(-\frac{i \pi}{4}\right) e_{\alpha+\beta}, & Q_{2}:=\sqrt{2} \exp \left(-\frac{i \pi}{4}\right) e_{-\alpha-\beta}, \\
\bar{Q}_{\dot{1}}:=\sqrt{2} \exp \left(-\frac{i \pi}{4}\right) e_{\beta}, & \bar{Q}_{\dot{2}}:=\sqrt{2} \exp \left(-\frac{i \pi}{4}\right) e_{-\beta} .
\end{array}
$$

They have the following commutation relations between themselves: 


$$
\begin{aligned}
& \left\{Q_{1}, Q_{1}\right\}=-i 2 \sqrt{2} e_{2 \alpha+2 \beta}=2\left(L_{13}-i L_{23}-L_{01}+i L_{02}\right), \\
& \left\{Q_{2}, Q_{2}\right\}=-i 2 \sqrt{2} e_{-2 \alpha-2 \beta}=2\left(L_{13}+i L_{23}-L_{01}-i L_{02}\right), \\
& \left\{Q_{1}, Q_{2}\right\}=-i 2\left(h_{\alpha}+h_{\beta}\right)=2\left(L_{03}+i L_{12}\right), \\
& \left\{\bar{Q}_{\dot{\eta}}, \bar{Q}_{\dot{\zeta}}\right\}=\left\{Q_{\zeta}, Q_{\eta}\right\}^{*} \quad\left(\bar{Q}_{\dot{\eta}}=Q_{\eta}^{*} \text { for } \eta=1,2 ; \dot{\eta}=\dot{1}, \dot{2}\right), \\
& {\left[Q_{1}, \bar{Q}_{\dot{1}}\right]=-i 2 e_{\alpha+2 \beta}=2\left(L_{04}+L_{34}\right),} \\
& \llbracket\left[Q_{1}, \bar{Q}_{\dot{2}}\right]=-i 2 e_{\alpha}=2\left(L_{14}-i L_{24}\right), \\
& {\left[\left[Q_{2}, \bar{Q}_{\dot{1}}\right]=-i 2(-1)^{\operatorname{deg} \alpha \cdot \operatorname{deg} \beta} e_{-\alpha}=2\left(L_{14}+i L_{24}\right),\right.} \\
& {\left[\left[Q_{2}, \bar{Q}_{\dot{2}}\right]=-i 2(-1)^{\operatorname{deg} \alpha \cdot \operatorname{deg} \beta} e_{-\alpha-2 \beta}=2\left(L_{04}-L_{34}\right) .\right.}
\end{aligned}
$$

Here $[\cdot, \cdot]] \equiv\{\cdot, \cdot\}$ for the $\mathbb{Z}_{2}$-case and $\left.[\cdot, \cdot]\right] \equiv[\cdot, \cdot]$ for the $\mathbb{Z}_{2} \times \mathbb{Z}_{2}$-case. Using the explicit formulas (28), (29), (32) and the commutation relations (26) we can also calculate commutation relations between the operators $L_{a b}$ and the supercharges $Q$ 's and $\bar{Q}$ 's .

\section{$4 \mathbb{Z}_{2}$ - and $\mathbb{Z}_{2} \times \mathbb{Z}_{2}$-graded Poincaré superalgebras}

Using the standard contraction procedure: $L_{\mu 4}=R P_{\mu}(\mu=0,1,2,3), Q_{\alpha} \rightarrow \sqrt{R} Q_{\alpha}$ and $\bar{Q}_{\dot{\alpha}} \rightarrow \sqrt{R} \bar{Q}_{\dot{\alpha}}(\alpha=1,2 ; \dot{\alpha}=1, \dot{2})$ for $R \rightarrow \infty$ we obtain the super-Poincaré algebra (standard and alternative) which is generated by $L_{\mu v}, P_{\mu}, Q_{\alpha}, \bar{Q}_{\dot{\alpha}}$ where $\mu, v=0,1,2,3 ; \alpha=1,2 ; \dot{\alpha}=1, \dot{2}$, with the relations (we write down only those which are distinguished in the $\mathbb{Z}_{2}$ - and $\mathbb{Z}_{2} \times \mathbb{Z}_{2}$-cases).

(I) For the $\mathbb{Z}_{2}$-graded Poincaré SUSY:

$$
\left[P_{\mu}, Q_{\alpha}\right]=\left[P_{\mu}, \bar{Q}_{\dot{\alpha}}\right]=0, \quad\left\{Q_{\alpha}, \bar{Q}_{\dot{\beta}}\right\}=2 \sigma_{\alpha \dot{\beta}}^{\mu} P_{\mu} .
$$

(II) For the $\mathbb{Z}_{2} \times \mathbb{Z}_{2}$-graded Poincaré SUSY:

$$
\left\{P_{\mu}, Q_{\alpha}\right\}=\left\{P_{\mu}, \bar{Q}_{\dot{\alpha}}\right\}=0, \quad\left[Q_{\alpha}, \bar{Q}_{\dot{\beta}}\right]=2 \sigma_{\alpha \dot{\beta}}^{\mu} P_{\mu},
$$

Let us consider the supergroups associated to the $\mathbb{Z}_{2^{-}}$and $\mathbb{Z}_{2} \times \mathbb{Z}_{2}$-graded Poincaré superalgebras. A group element $g$ is given by the exponential of the superPoincaré generators, namely

$$
g\left(x^{\mu}, \omega^{\mu v}, \theta^{\alpha}, \bar{\theta}^{\dot{\alpha}}\right)=\exp \left(x^{\mu} P_{\mu}+\omega^{\mu v} M_{\mu v}+\theta^{\alpha} Q_{\alpha}+\bar{Q}_{\dot{\alpha}} \bar{\theta}^{\dot{\alpha}}\right) .
$$

Because the grading of the exponent is zero $((0)$ or $(00))$ and the result is as follows. 
1). $\mathbb{Z}_{2}$-case: $\operatorname{deg} P=\operatorname{deg} x=0, \operatorname{deg} Q=\operatorname{deg} \bar{Q}=\operatorname{deg} \theta=\operatorname{deg} \bar{\theta}=1$. This means that

$$
\left[x_{\mu}, \theta_{\alpha}\right]=\left[x_{\mu}, \bar{\theta}_{\dot{\alpha}}\right]=\left\{\theta_{\alpha}, \bar{\theta}_{\dot{\beta}}\right\}=\left\{\theta_{\alpha}, \theta_{\beta}\right\}=\left\{\bar{\theta}_{\dot{\alpha}}, \bar{\theta}_{\dot{\beta}}\right\}=0 .
$$

2). $\mathbb{Z}_{2} \times \mathbb{Z}_{2}$-case: $\operatorname{deg} P=\operatorname{deg} x=(11), \operatorname{deg} Q=\operatorname{deg} \theta=(10), \operatorname{deg} \bar{Q}=\operatorname{deg} \bar{\theta}=$ (01). This means that

$$
\left\{x_{\mu}, \theta_{\alpha}\right\}=\left\{x_{\mu}, \bar{\theta}_{\dot{\alpha}}\right\}=\left[\theta_{\alpha}, \bar{\theta}_{\dot{\beta}}\right]=\left\{\theta_{\alpha}, \theta_{\beta}\right\}=\left\{\bar{\theta}_{\dot{\alpha}}, \bar{\theta}_{\dot{\beta}}\right\}=0 .
$$

One defines the superspaces as the coset spaces of the standard and alternative superPoincaré groups by the Lorentz subgroup, parameterized the coordinates $x^{\mu}, \theta^{\alpha}$, $\bar{\theta}^{\dot{\alpha}}$, subject to the condition $\bar{\theta}^{\dot{\alpha}}=\left(\theta^{\alpha}\right)^{*}$. We can define a superfield $\mathscr{F}$ as a function of superspace.

Acknowledgements The author would like to thank the Organizers for the kind invitation to speak at the 10-th International Workshop "Lie Theory and Its Applications in Physics" (LT-10, Varna, June 17-23, 2013), and for support of his visit on the Workshop. The paper was supported by the RFBR grant No.11-01-00980-a and the grant No.12-09-0064 of the Academic Fund Program of the National Research University Higher School of Economics.

\section{References}

1. V.G. Kac. Lie superalgebras. Adv. Math. 26, (1977) 8-96.

2. L. Frappat, A. Sciarrino, P. Sorba, Dictinary on Lie supealgebras. arXiv:hep-th/9607161v1

3. J. Lukierski, V. Rittenberg. Color-de Sitter and color-conformal superalgebras. Phys. Rev. D 18, No.2 (1978) 385-389.

4. V. Rittenberg, D. Wyler. Sequences of $\mathbb{Z}_{2} \times \mathbb{Z}_{2}$-graded Lie algebras and superalgebras. J. Math. Phys. 19, No.10 (1978) 2193-2200.

5. V. Rittenberg, D. Wyler. Generalized superalgebras. Nucl. Phys. 139B, No.10 (1978) 189200.

6. M. Sheunert. Generalized Lie algebras. J. Math. Phys. 20(4), (1979) 712-720.

7. M.A. Vasiliev. De Sitter supergravity with positive cosmological constant and generalized Lie superalgebras. Class. Quantum Grav. 2, (1985) 645-659.

8. A.A. Zheltukhin. Para-Grassmann extension of the Neveu-Schwarz-Ramond algebra. Teor. Mat. Fiz. 71, No. 2, (1987) 218-225. 